\title{
Model-Based Fault Detection and Isolation Method Using ART2 Neural Network
}

\author{
I. S. Lee, ${ }^{1, *}$ J. T. Kim, ${ }^{2, * *}$ J. W. Lee, ${ }^{2, \dagger}$ D. Y. Lee, ${ }^{2, \ddagger}$ K. Y. Kim ${ }^{3, \S}$ \\ ${ }^{1}$ School of Electronics and Electrical Engineering, Sangju National University, \\ 386, Gajang-Dong, Sangju-Si, Kyungpook, 742-711, Korea \\ ${ }^{2}$ Man-Machine Interface Team, Korea Atomic Energy Research Institute, \\ P.O. Box 105, Daejon, 305-600, Korea \\ ${ }^{3}$ Department of Electrical and Electronic Engineering, Cheju National \\ University, Cheju, 690-756, Korea
}

\begin{abstract}
This article presents a model-based fault diagnosis method to detect and isolate faults in the robot arm control system. The proposed algorithm is composed functionally of three main parts: parameter estimation, fault detection, and isolation. When a change in the system occurs, the errors between the system output and the estimated output cross a predetermined threshold, and once a fault in the system is detected, the estimated parameters are transferred to the fault classifier by the adaptive resonance theory 2 neural network (ART2 NN) with uneven vigilance parameters for fault isolation. The simulation results show the effectiveness of the proposed ART2 NN-based fault diagnosis method. (C) 2003 Wiley Periodicals, Inc.
\end{abstract}

\section{INTRODUCTION}

Fault diagnosis of industrial processes becomes more important in light of increased automation in industry. The early detection and isolation of faults can help avoid major system breakdowns.

There have been many methods for fault detection and isolation (FDI) of the system. The model-based FDI methods rely on the idea of analytic redundancy. These methods fall into two major groups: (1) state estimation-based methods and (2) parameter estimation-based methods. The state estimation-based methods are not easy applied to the complex system because the state space model is not easy to obtain, ${ }^{1}$ whereas, the model-based FDI methods through parameter estimation are effectively used in complex systems because the input/output model is easy to

\footnotetext{
*Author to whom all correspondence should be addressed: e-mail: islee@sangju.ac.kr.

**e-mail: jtkim@kaeri.re.kr.

†e-mail: leejw@kaeri.re.kr.

†-mail: dylee2@kaeri.re.kr.

§e-mail: kyungyk@cheju.ac.kr.
}

INTERNATIONAL JOURNAL OF INTELLIGENT SYSTEMS, VOL. 18, 1087-1100 (2003) (C) 2003 Wiley Periodicals, Inc. Published online in Wiley InterScience (www.interscience.wiley.com). • DOI 10.1002/int.10134 
obtain. ${ }^{1}$ This approach is based on the assumption that the faults are reflected in the physical system parameters such as resistances, capacities, etc. Many input/output model-based methods are developed. ${ }^{2-5}$ In these methods, the system parameters are estimated and the error between the system output and the estimated output is used for fault detection. The estimated parameters are used to compute the actual physical parameters from the relationship between model coefficients and actual physical parameters. However, in practice the mathematical description of the relationship between model parameters and physical parameters is not easy to obtain because of the nonlinearities. ${ }^{5}$ This limitation can be overcome by a pattern recognition method. ${ }^{6}$ However, it is difficult to isolate new unencountered faults.

Srinivasan et al..$^{5}$ proposed an FDI algorithm using the Hopfield and adaptive resonance theory 1 neural network (ART1 NN). In this method, the algorithm is composed functionally of three main parts: the estimation part for estimation of system parameters, the fault detection part, and the fault isolation part by ART1 NN. However, the ART1 NN is used for classification of the binary patterns. Therefore, ART2 NN is suitable for classification because the estimated parameters are analog patterns. Usually, the estimated parameters have widely varying magnitudes. Therefore, the parameter with a large magnitude will greatly affect the classification result as compared with a parameter with a smaller magnitude. For this reason, when the conventional ART2 $\mathrm{NN}^{7,8}$ is used for the fault isolation, the isolation accuracy deterioration of the classifier occurs.

In this article, we propose a fault diagnosis method using ART2 NN with uneven vigilance parameters to detect and isolate system faults and sensor bias faults in the robot arm control system. ${ }^{9}$ The proposed algorithm is based on ART2 NN with uneven vigilance parameters. ${ }^{10}$ Because ART2 NN, with uneven vigilance parameters, is an unsupervised neural network, the proposed fault classifier does not require the knowledge of all possible faults to isolate the faults that occurred in the system. In particular, the proposed fault classifier can isolate the fault more flexibly because it uses uneven vigilance parameters to classify the fault patterns. Simulations are performed to evaluate the performance of the proposed model-based fault diagnosis method for the robot arm control system.

The remainder of this article is organized as follows. In Section 2, we propose a fault diagnosis method using ART2 NN with uneven vigilance parameters. In Section 4, simulations are presented to show the proposed fault diagnosis method. The last section draws conclusions.

\section{FDI METHOD USING ART2 NN}

In this section, an FDI method based on the ART2 NN is presented. The structure of the proposed method is the same as that of the conventional method ${ }^{5}$ except for the parameter estimation and isolation methods (Figure 1). The algorithm contains three main steps: a parameter estimation part, a fault detection part by threshold test, and a fault isolation part by fault classifier. In the proposed method, the ART2 NN with uneven vigilance parameters is used for isolation. Also, both the system faults (component faults) and sensor bias faults can be isolated. In the next four sections, the individual steps are described. 
Fault Classifier using ATR2 NN

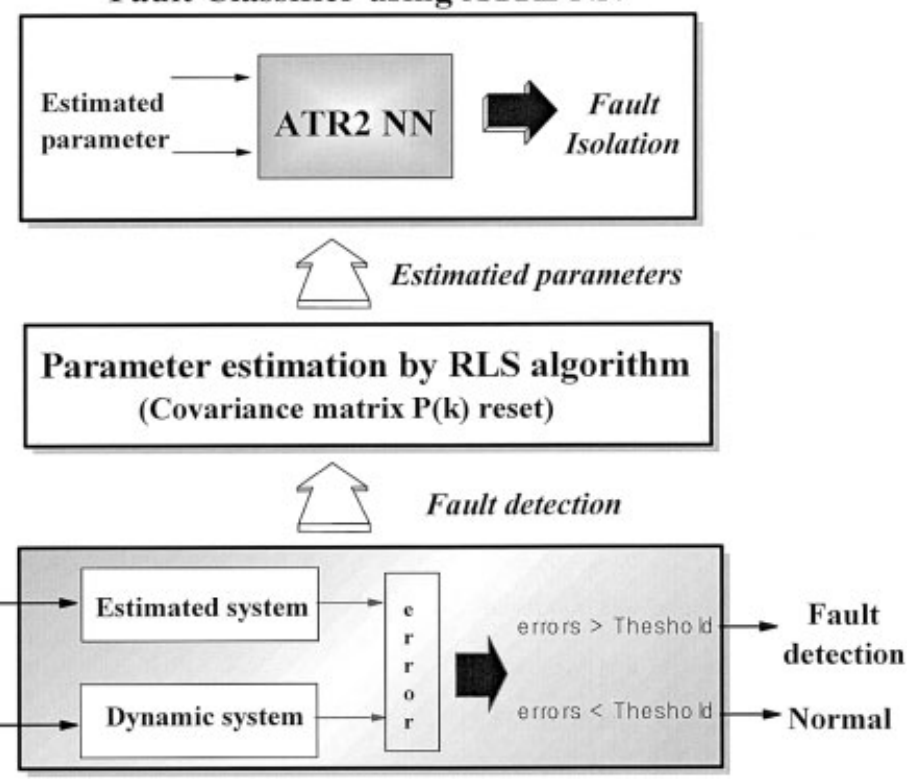

Figure 1. Structure of the FDI algorithm.

\subsection{Estimation of System Parameters}

Consider a single-input single-output discrete-time linear system,

$$
\begin{aligned}
y(k)=a_{1} y(k-1)+\cdots+a_{n} y(k-n)+b_{1} u(k-1) & \\
& +\cdots+b_{m} u(k-m)+\varepsilon(k)
\end{aligned}
$$

where $y(k)$ and $u(k)$ are the output and the input at time $k$, respectively, and $\varepsilon(k)$ is the white noise.

The system model can be written as follows:

$$
y(k)=\boldsymbol{\theta}^{T} \boldsymbol{\phi}(k-1)+\varepsilon(k)
$$

where $\boldsymbol{\theta}=\left[\begin{array}{lll}a_{1} \cdots a_{n} & b_{1} \cdots b_{n}\end{array}\right]^{T}$,

$$
\boldsymbol{\phi}(k-1)=[y(k-1) \cdots y(k-n) u(k-1) \cdots u(k-m)]^{T}
$$

and $\boldsymbol{\theta}$ and $\boldsymbol{\phi}(k-1)$ are the system parameter vector and measurement vector, respectively.

The system parameters $\boldsymbol{\theta}$ are mostly more or less intricate relationships of several physical parameters, e.g. resistances, capacitances. Therefore, the system faults (component faults) could be expressed as a change in the system parameters. When the system has all possible system and sensor offset faults, the system model can be modeled by

$$
y(k)=\boldsymbol{\theta}^{T} \boldsymbol{\phi}(k-1)+\Delta \boldsymbol{\theta}^{T} \boldsymbol{\phi}(k-1)+f_{s}+\varepsilon(k)
$$




$$
=\boldsymbol{\theta}_{f}^{T} \boldsymbol{\phi}(k-1)+f_{s}+\varepsilon(k)
$$

where $f_{s}$ is the additive sensor bias. Therefore, estimated parameters $\hat{\boldsymbol{\theta}}_{f}$ and estimated sensor bias $\hat{f}_{s}$ are used to classify faults.

Let the cost function, output error $e(k)$, and estimated output $\hat{y}(k)$ be defined as

$$
\begin{gathered}
J(k)=\sum_{i=1}^{k} e^{2}(i) \\
e(k)=y(k)-\hat{y}(k) \\
\hat{y}(k)=\hat{\boldsymbol{\theta}}_{\text {aug }}^{T}(k-1) \boldsymbol{\phi}_{\mathrm{aug}}(k-1)
\end{gathered}
$$

where

$$
\begin{gathered}
\hat{\boldsymbol{\theta}}_{\text {aug }}=\left[\begin{array}{llll}
\hat{a}_{1} \cdots \hat{a}_{n} & \hat{b}_{1} \cdots \hat{b}_{n} & \hat{f}_{s}
\end{array}\right]^{T} \\
\boldsymbol{\phi}_{\mathrm{aug}}(k-1)=\left[\begin{array}{lll}
y(k-1) \cdots y(k-n) & u(k-1) \cdots u(k-m) & 1
\end{array}\right]^{T}
\end{gathered}
$$

and $\hat{\boldsymbol{\theta}}_{\text {aug }}$ and $\boldsymbol{\phi}_{\text {aug }}$ are augmented parameter vector and augmented measurement vector, respectively.

An estimate $\hat{\boldsymbol{\theta}}_{\text {aug }}$, minimizing the cost function in Equation 4 is given by the following recursive least square (RLS) algorithm ${ }^{11}$ :

$$
\begin{gathered}
\hat{\boldsymbol{\theta}}_{\mathrm{aug}}(k)=\hat{\boldsymbol{\theta}}_{\mathrm{aug}}(k-1)+L(k) e^{0}(k) \\
e^{0}(k)=y(k)-\boldsymbol{\phi}_{\mathrm{aug}}^{T}(k-1) \hat{\boldsymbol{\theta}}_{\mathrm{aug}}(k-1) \\
L(k)=\frac{P(k-1) \boldsymbol{\phi}_{\mathrm{aug}}(k-1)}{1+\boldsymbol{\phi}_{\mathrm{aug}}^{T}(k-1) P(k-1) \boldsymbol{\phi}_{\mathrm{aug}}(k-1)} \\
\mathbf{P}(k)=\mathbf{P}(k-1)-\frac{\mathbf{P}(k-1) \boldsymbol{\phi}_{\mathrm{aug}}(k-1) \boldsymbol{\phi}_{\mathrm{aug}}^{T}(k-1) \mathbf{P}(k-1)}{1+\boldsymbol{\phi}_{\mathrm{aug}}^{T}(k-1) \mathbf{P}(k-1) \boldsymbol{\phi}_{\mathrm{aug}}(k-1)}
\end{gathered}
$$

where $\mathbf{P}(k)$ is the covariance matrix of $\hat{\boldsymbol{\theta}}_{\text {aug }}$.

\subsection{Fault Detection}

Comparing the output of the system with the estimated output generates errors. Fault is detected when the errors exceed a predetermined threshold value.

Fault is detected by the following threshold test:

$$
J_{L}(k)=\sum_{i=k-L+1}^{k} e^{2}(i)>\delta_{f}
$$

where $e$ is the error between the system output and the estimated output, $L$ is the moving window length, and $\delta_{f}$ is the predetermined threshold for fault detection. The 
big size of the window $L$ will improve the performance of the estimation, but the algorithm is insensitive to the fault. On the other hand, window $L$ with small values will be sensitive to the fault. But the performance of the estimation is decreased and it may cause a missed isolation. Therefore, window length $L$ should be selected properly.

If the estimated parameters converge to the system parameter, then the error between the system output and estimate output has a similar property of the system noise. ${ }^{12}$ Thus, the error $e$ has a normal distribution $\mathrm{N}\left(0, \sigma^{2}\right)$. Also, the sum of the normalized square errors in the moving window has a $\chi_{L}^{2}$-distribution with $L$ degrees of freedom as follows:

$$
\overline{J_{L}}(k)=\sum_{i=k-L+1}^{k} \frac{e^{2}(i)}{\sigma^{2}} \sim \chi_{L}^{2}
$$

If the false-alarm probability limit is $\alpha$,

$$
\operatorname{Pr}\left(\sum_{i=k-L+1}^{k} \frac{e^{2}(i)}{\sigma^{2}}>\delta^{O}\right)=\alpha
$$

and the threshold is obtained as

$$
\delta_{f}=\sigma^{2} \delta^{O}
$$

Usually, the change in the system parameters by fault is the larger than the system noise. Therefore, false-alarm probability is considered to select the threshold and is calculated from Equations 15 and 16.

If a fault corresponds to small changes in the system parameters, the missfault detection probability increases. Hence, the heuristic knowledge about the system is necessary in determining the threshold.

Once a fault is detected, the varied system parameters are estimated. However, the covariance matrix $\mathbf{P}(k)$ of the RLS algorithm may reduce rapidly. This makes the estimation algorithm slow to sudden changes in the system. To overcome this problem, the covariance matrix resetting method is considered. The judgment on the instance at which the estimated parameters converge to system parameter is made through the following convergence test:

$$
J_{L}(k)=\sum_{i=k-L+1}^{k} e^{2}(i)<\delta^{*}
$$

where $\delta^{*}$ is the convergence threshold.

\subsection{Fault Isolation by ART2 NN with Uneven Vigilance Parameters}

The estimated system parameters are used to classify faults. Architecture of the ART2 NN is shown in Figure 2. The proposed ART2 NN with uneven vigilance parameters has the same architecture as the general ART2 NN. ${ }^{7}$ How- 


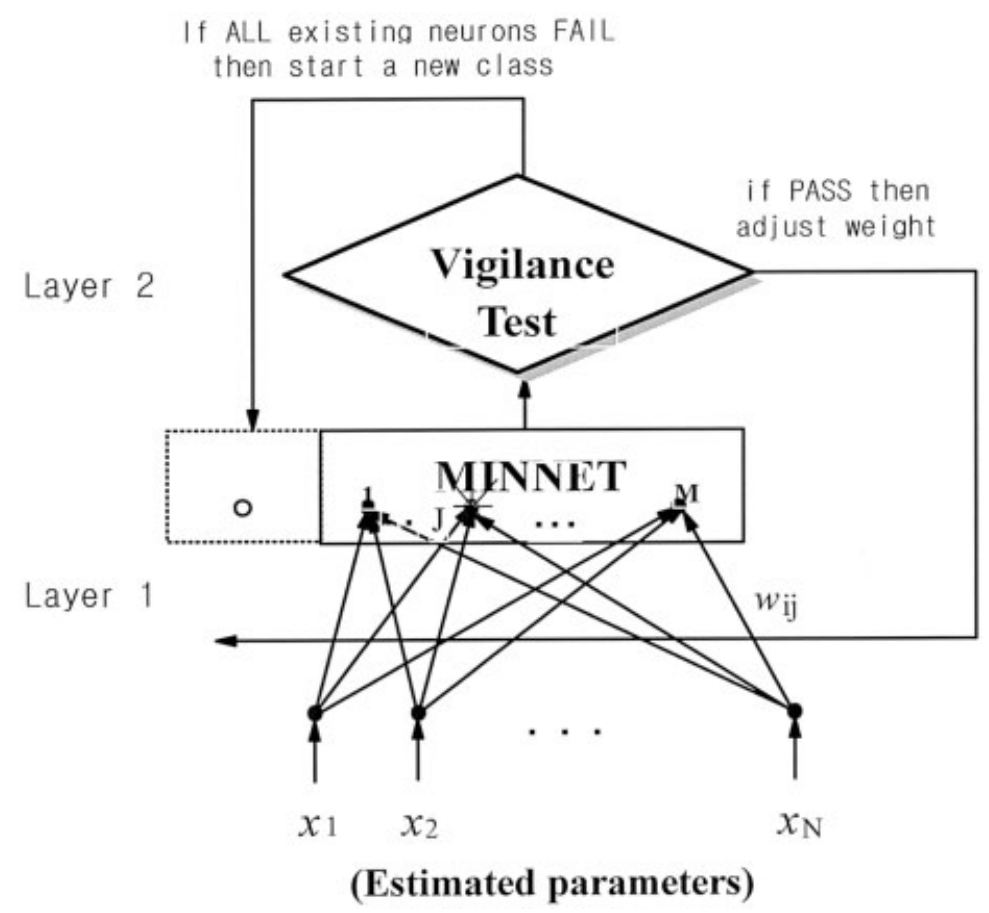

Figure 2. Architecture of the ART2 NN.

ever, in the proposed neural network, a new vigilance test is used to classify the input patterns. follows:

The distance between the input patterns and $j$ th output node is computed as

$$
d_{j}=\left\|W_{j}-X\right\|_{\infty}^{E} \underline{\Delta} \max _{i}\left|\frac{1}{\varepsilon_{i}}\left(w_{i j}-x_{i}\right)\right|, \quad j=1,2, \ldots, M
$$

where $x_{i}$ is the input of the input node $i, i=1,2, \ldots, N, N$ is the number of input nodes, $w_{i j}$ is the weight from output node $j$ to input node, $M$ is the number of the output nodes (created classes), $\|\cdot\|_{\infty}^{E}$ is the weighted infinite norm, $\mathbf{E}=\operatorname{diag}[(1 /$ $\left.\left.\varepsilon_{1}\right),\left(1 / \varepsilon_{2}\right), \cdots\left(1 / \varepsilon_{N}\right)\right]$ is the $N \times N$ diagonal weighted matrix, and $\varepsilon_{i}$ is the $i$ th vigilance parameter for $i$ th input node. To improve the classification accuracy, the vigilance parameter for the parameter with a large magnitude variation is selected large. On the other hand, the vigilance parameter for the parameter with a small magnitude variation is selected small.

If the distance between the input patterns and the $J$ th output node is minimum, then class $J$ is selected as the winner node. Verification is done whether input pattern $X$ really belongs to the winner class $J$ by performing the vigilance test as follows: 


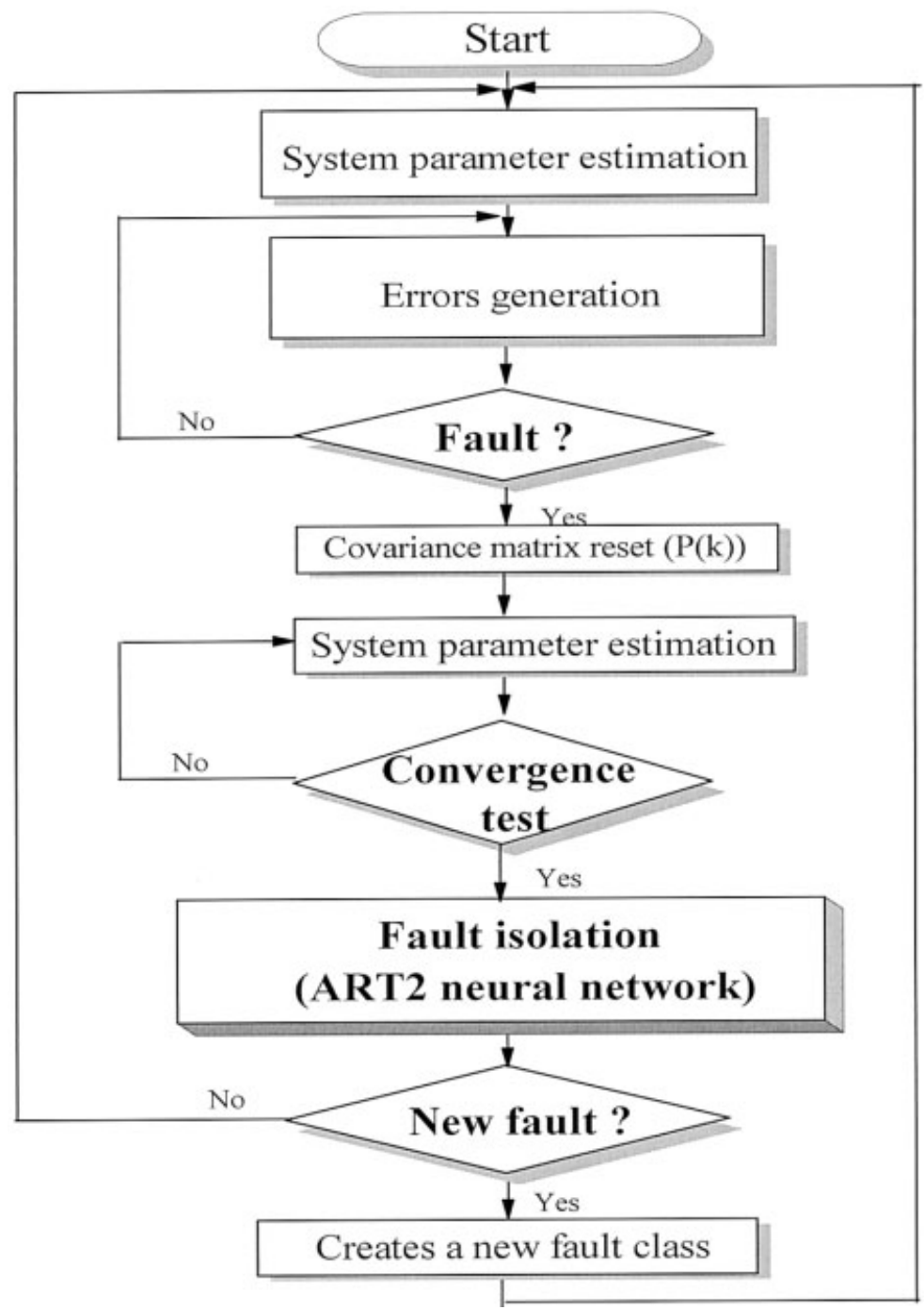

Figure 3. Flowchart of the fault diagnosis algorithm.

vigilance test condition: $\left\|W_{J}-X\right\|_{\infty}^{E}<1$

If the winner class $J$ passes the vigilance test, adjust the weights of the class $J, W_{J}$ by

$$
W_{J}^{\text {new }}=\frac{X+W_{J}^{\text {old }}\left[\operatorname{class}_{J}^{\text {old }}\right]}{\left[\operatorname{class}_{J}^{\text {old }}\right]+1}
$$

where $\left[\right.$ class $\left._{i}\right]$ is the number of the patterns in class $i$.

On the other hand, if the class $J$ fails the vigilance test, a new class (output node) is created with weight $W_{M+1}=X$. 


\subsection{Fault Diagnosis Procedures}

A flowchart of the FDI algorithm is shown in Figure 3. This algorithm procedure is summarized as follows:

Step 1. System parameters are estimated by the RLS algorithm.

Step 2. When a change in the system occurs, the sum of the square errors between the system output and the estimated output cross a predetermined threshold and fault is detected. If fault is detected, then varied system parameters are estimated. In this study, we assumed that fault has not occurred at system start-up. Therefore, the deviation in parameter estimates during start-up is regarded as a normal state. Step 3. The estimated parameters are transferred to the fault classifier by ART2 $\mathrm{NN}$ with uneven vigilance parameters for fault isolation.

Step 4. When existing fault has occurred, the fault classifier gives the fault class number (ART2 NN output) of an existing fault. On the other hand, in case new fault has occurred, the ART2 NN creates a new fault class.

\section{SIMULATION RESULTS}

Simulations are performed to evaluate the performance of the ART2 NNbased FDI algorithm. The transfer function of the robot arm control system is given as

$$
\frac{\boldsymbol{\theta}_{o}(s)}{\boldsymbol{\theta}_{i}(s)}=\frac{\mathbf{E} s+F}{s^{4}+A s^{3}+B s^{2}+C s+D}
$$

where

$$
\begin{aligned}
& A=\frac{J_{L}\left(B_{m}+B_{B}\right)+J_{m}\left(B_{L}+B_{B}\right)}{A_{1}} \\
& B=\frac{B_{L} B_{m}+B_{B}\left(B_{L}+B_{m}\right)+K\left(J_{m}+J_{L}\right)}{A_{1}} \\
& C=\frac{K\left(B_{L}+B_{m}\right)+K_{i} K_{p} B_{B}}{A_{1}}, \quad D=\frac{K K_{i} K_{p}}{A_{1}} \\
& E=\frac{K_{i} K_{p} B_{B}}{A_{1}}, \quad F=\frac{K K_{i} K_{p}}{A_{1}}
\end{aligned}
$$

The $K_{p}$ is the $P$ controller constant; and $K, K_{i}$, and $B_{B}$ are the spring constant, torque constant, and shaft viscous friction coefficient between the robot arm and the motor, respectively. The $J_{m}$ and $B_{m}$ represent the inertia of the motor and viscous friction coefficient, respectively. The $J_{L}$ and $B_{L}$ are the inertia of the load (robot arm) and viscous friction coefficient, respectively, and $A_{1}=J_{m} J_{L}$. Parameter values for simulation are as follows:

$$
K=100, \quad K_{i}=0.4, \quad B_{B}=0.2
$$




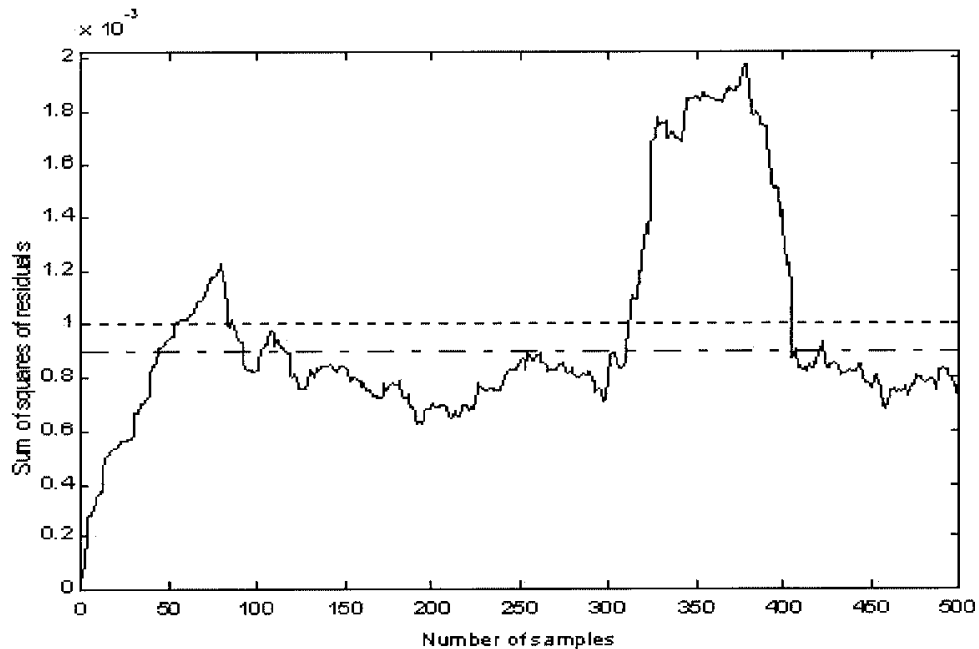

4 (a)

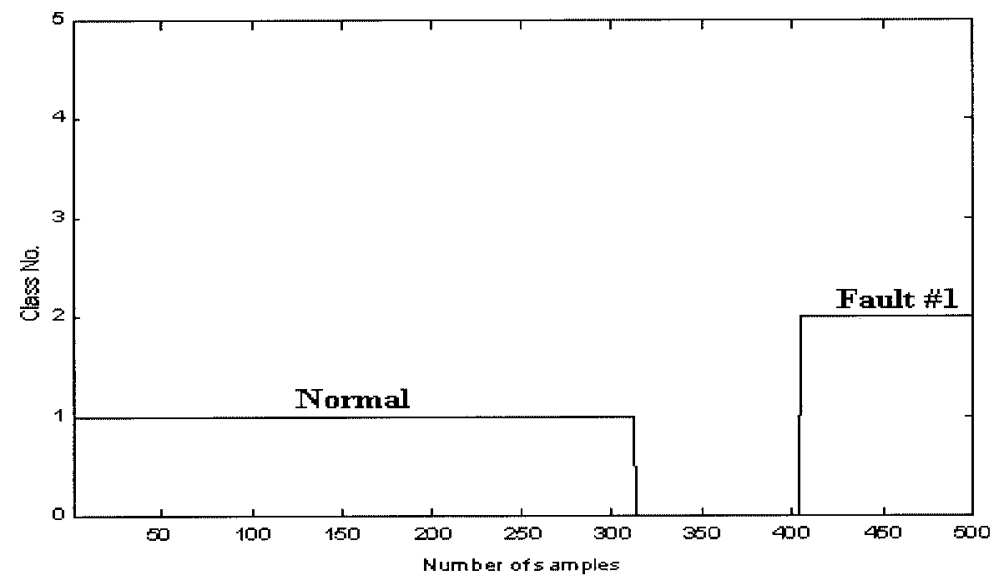

4 (b)

Figure 4. Results of detection and isolation for Fault 1. (a) Change of $J_{L}$ and fault detection (dashed line; $\delta_{f}$; dashdot line, $\delta^{*}$ ); (b) classification result by proposed classifier.

$$
J_{m}=0.2, \quad B_{m}=0.25, \quad J_{L}=0.6, \quad B_{L}=0.01
$$

The sampled input-output system can be described by a fourth-order discrete time system as follows:

$$
\begin{aligned}
\boldsymbol{\theta}_{o}(k+1)=a_{1} \boldsymbol{\theta}_{o}(k)+a_{2} \boldsymbol{\theta}_{o}(k-1) & +a_{3} \boldsymbol{\theta}_{o}(k-2)+a_{4} \boldsymbol{\theta}_{o}(k-3)+b_{1} \boldsymbol{\theta}_{i}(k) \\
+ & b_{2} \boldsymbol{\theta}_{i}(k-1)+b_{3} \boldsymbol{\theta}_{i}(k-2)+b_{4} \boldsymbol{\theta}_{i}(k-3)
\end{aligned}
$$

where $a_{1}, a_{2}, a_{3}, a_{4}, b_{1}, b_{2}, b_{3}$, and $b_{4}$ are the system parameters to be estimated and are used for fault isolation; sampling time is 0.05 seconds and output mea- 


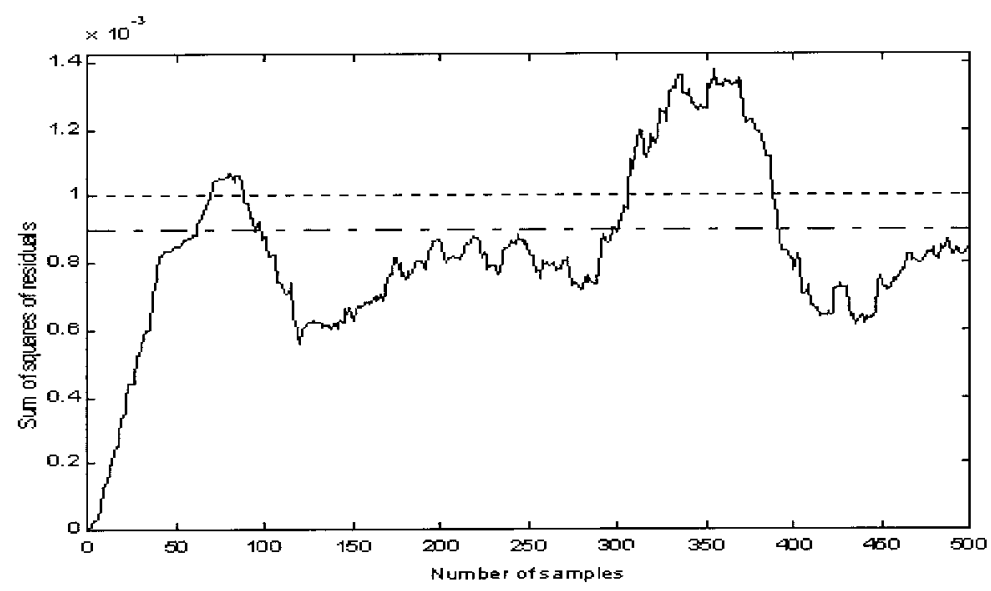

$5(a)$

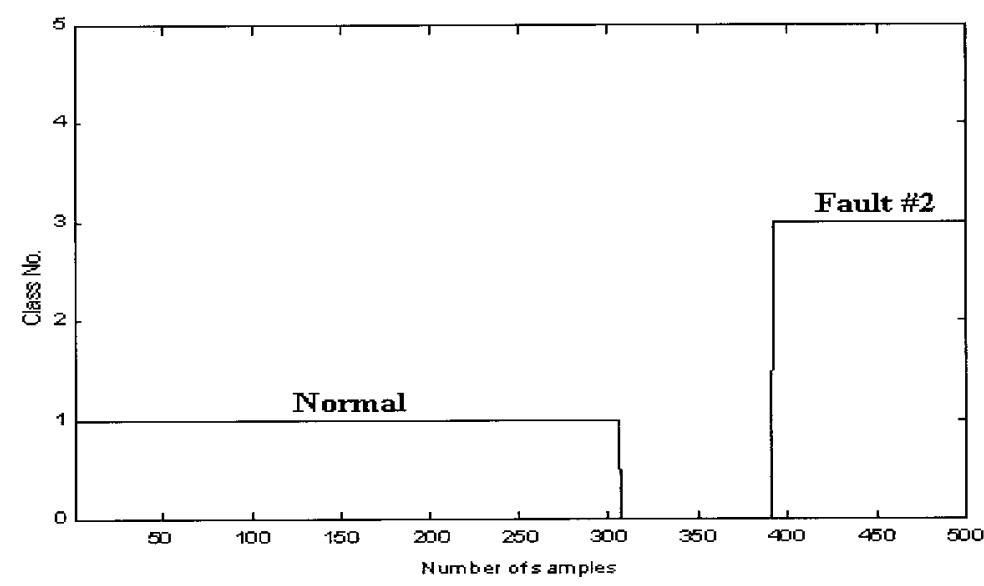

$5(\mathrm{~b})$

Figure 5. Results of detection and isolation for Fault 2. (a) Change of $J_{L}$ and fault detection (dashed line, $\delta_{f}$; dashdot line, $\delta^{*}$ ); (b) classification result by proposed classifier.

surement noise is white noise with variance $\sigma^{2}=0.9 * 10^{-5}$. Here, we choose $\alpha=$ $0.01(1 \%)$ and the moving window length $L=30$, threshold $\delta_{f}=0.01$ (from Equations 15 and 16), and convergence threshold $\delta^{*}$ is set to 0.0009 . We choose the vigilance parameters of ART2 NN with uneven vigilance parameters as $\varepsilon_{1}=$ $\varepsilon_{2}=\varepsilon_{3}=\varepsilon_{4}=0.2, \varepsilon_{5}=\varepsilon_{6}=\varepsilon_{7}=\varepsilon_{8}=0.02$.

To verify the proposed diagnosis algorithm, four types of faults are introduced to the system at the 300th sample number. The following faults are simulated:

Fault 1. Increased inertia of the robot arm $\left(J_{L}\right)$

Fault 2. Reduced spring constant $(K)$ 


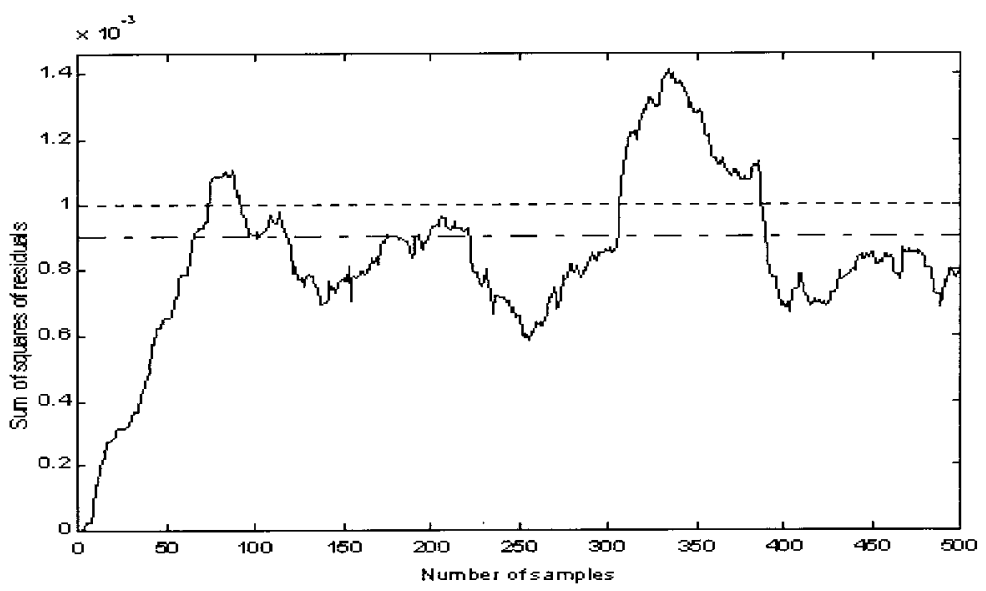

$6(\mathbf{a})$

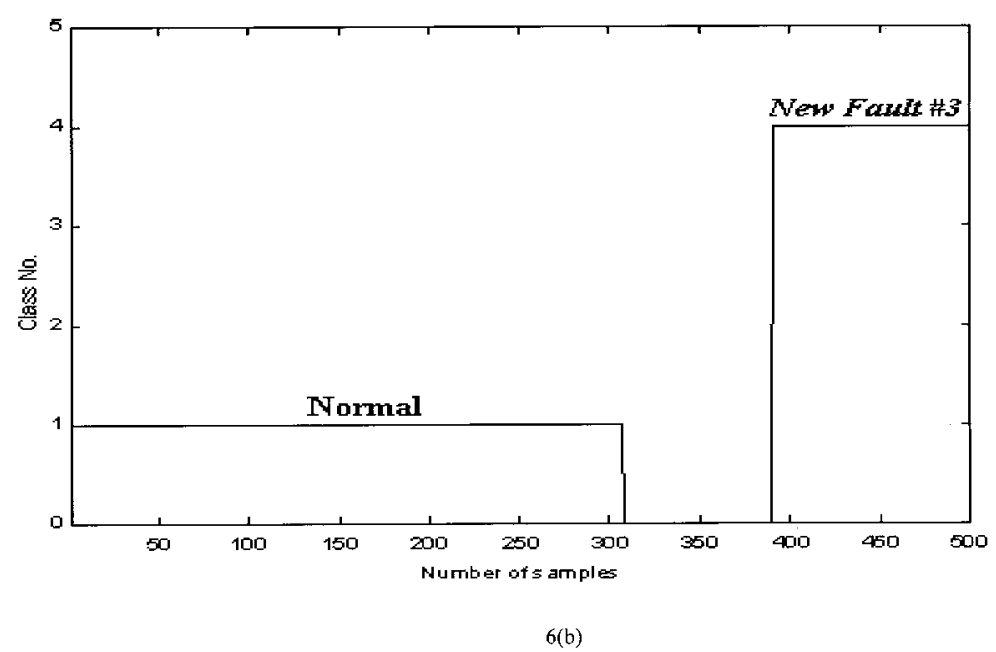

Figure 6. Results of detection and isolation for the new fault (Fault 3). (a) Change of $J_{L}$ and fault detection (dashed line, $\delta_{f}$; dashdot line, $\delta^{*}$ ); (b) classification result by proposed classifier.

Fault 3. Both Faults 1 and 2

Fault 4. Sensor bias $\left(f_{s}=0.5\right)$ exists but system is in normal state

The simulation results for the Faults 1, 2, 3, and 4 are shown in Figures 4-7, respectively. Figures 4(b) and 5(b) show the classification results after three faults classes (i.e., Class 1 for normal state, Class 2 for Fault 1, and Class 3 for Fault 2) are generated. Figure 6 shows the results that the new Fault 3 occurs at the 300th sample number. Figure 4(a) shows the variations of the sum of squares of errors in the moving window (fault occurs at the 300th sample number), and Figure 4(b) shows the output of ART2 NN with uneven vigilance parameters. The simulation 


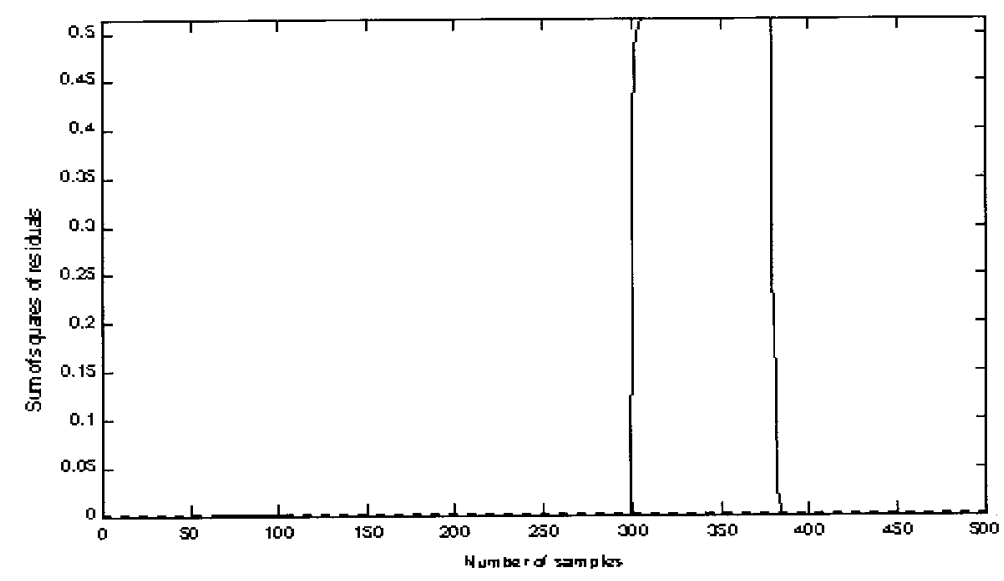

$7(a)$

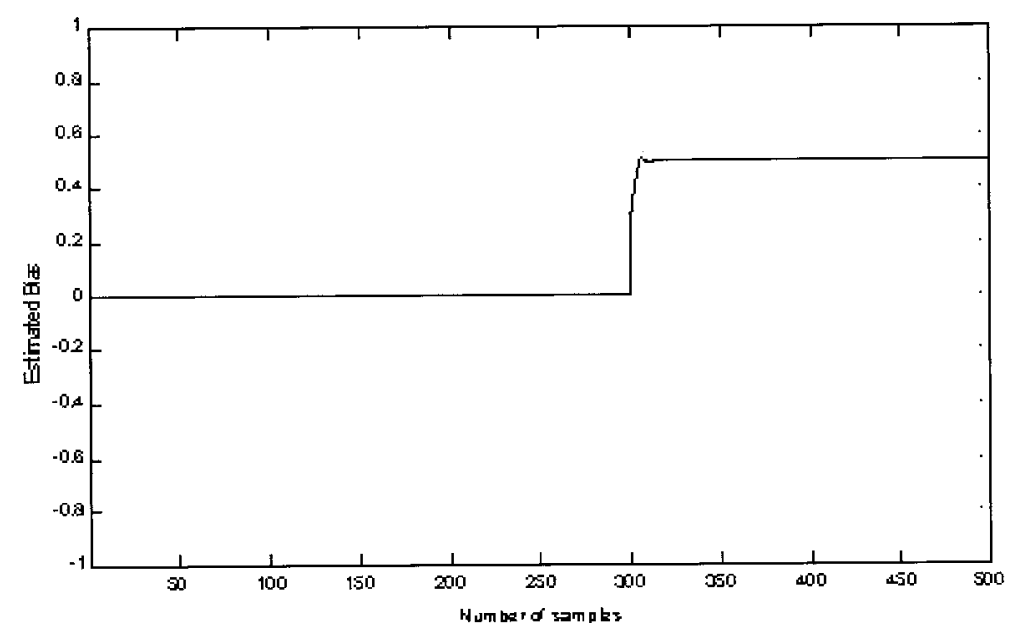

$7(b)$

Figure 7. Results of detection and isolation for the new fault (Fault 4). (a) Change of $J_{L}$ and fault detection (dashed line, $\delta_{f}$; dashdot line, $\delta^{*}$ ); (b) estimated sensor bias; (c) classification result by proposed classifier.

results showed that the proposed classifier successfully classifies the fault as Class 2. In addition, the results shown in Figure $5(\mathrm{a}, \mathrm{b})$ show that the fault classifier by the proposed neural network classifies the fault well.

Figure 6 shows the results of detection and isolation for new Fault 3. From the results, we can see that ART2 NN successfully creates a new fault Class 4 . The results of detection and isolation for Fault 4 are shown in Figure 7. From the classification and estimated sensor bias results, we know that system is a normal state but a sensor bias fault exists. 


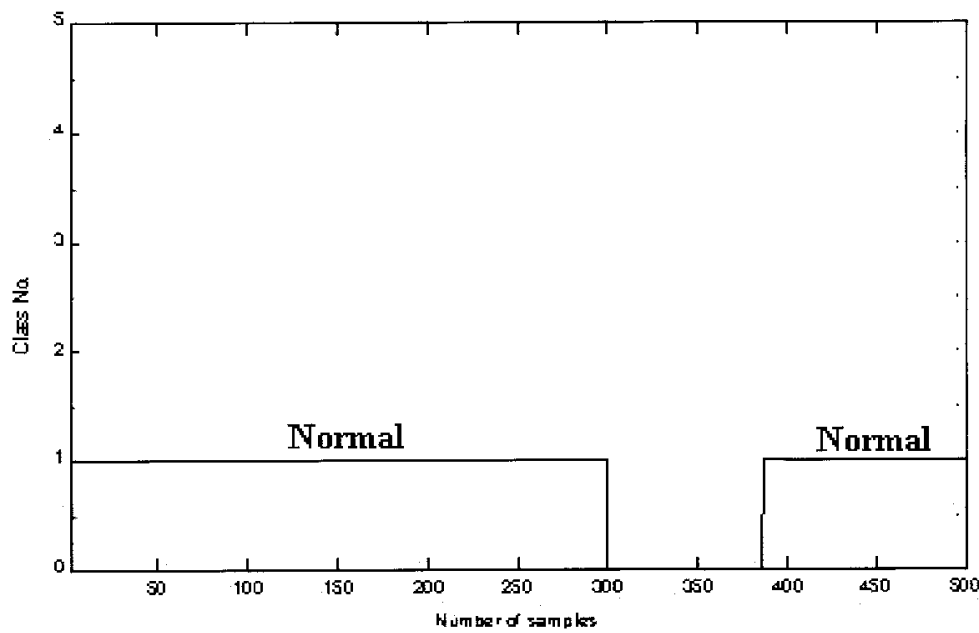

7(c)

Figure 7 (continued)

\section{CONCLUSIONS}

In this study, we proposed a model-based detection and isolation algorithm using ART2 NN with uneven vigilance parameters to detect and isolate system faults and sensor bias faults in linear systems. Because ART2 NN is an unsupervised neural network, it can adaptively learn and classify input patterns without a priori knowledge of classes. Therefore, the fault classifier does not require the knowledge of all possible faults to isolate the faults that occurred in the system. Also, the proposed fault classifier can isolate the fault more precisely because it uses uneven vigilance parameters to classify the fault patterns. From the computer simulation results, the proposed model-based fault diagnosis algorithm, using ART2 NN with uneven vigilance parameters, was applied successfully to the FDI problem in the robot arm control system.

\section{Acknowledgments}

This work was supported by the Nuclear Academic Research Program of the Ministry of Science and Technology (MOST), Korea.

\section{References}

1. Smyth P. Hidden markov models for fault detection in dynamic systems. Pattern Recognition 1994;27(1):149-164.

2. Isermann R. Model based fault detection and diagnosis methods. Proc ACC 1995;16051609.

3. Patton R, Frank P, Clark R. Fault diagnosis in dynamic systems theory and application. Upper Saddle River, NJ: Prentice Hall; 1989. 
4. Chen J, Patton R. Robust model-based fault diagnosis for dynamic systems. Dordrecht, The Netherlands: Kluwer Academic Publishers; 1999.

5. Srinivasan A, Batur C. Hopfield/ART-1 neural network-based fault detection and isolation. IEEE Trans Neur Netw 1994;5(6):890-899.

6. Polycarpou MM, Vemuri AT. Learning methodology for failure detection and accommodation. IEEE Contr Syst Mag 1995;15(3):16-24.

7. Kung SY. Digital neural networks. Upper Saddle River, NJ: Prentice Hall; 1993.

8. Pao YH. Adaptive pattern recognition and neural networks. Reading, MA: AddisonWesley; 1989.

9. Lee IS, Kim JT, Lee JW, Lee DY, Lee YJ, Kim KY. Model-based fault detection and isolation method using ART2 neural network. Presented at the 21st IASTED Int Conf on Modelling, Identification and Control (MIC 2002), Innsbruck, Austria, 2002. pp 186-191.

10. Lee IS, Shin PL, Jeon GJ. Multiple faults diagnosis of a linear system using ART2 neural networks. J Control Automation Syst Eng 1997;3(3):244-251.

11. Ljung L, Soderstrom T. Theory and practice of recursive identification. Cambridge, MA: MIT Press; 1983.

12. Cryer JD. Time series analysis. N. Scituale, MA: Duxbury Press; 1986. 